\title{
Comportamientos de ciudadanía organizacional como antecedente de la cultura, compromiso y satisfacción laboral en empresas de servicio
}

\author{
Celia Estephanie Martínez-Correa \\ al137278@alumnos.uacj.mx \\ Michelle López-Valdez \\ al149632@alumnos.uacj.mx
}

\author{
María Marisela Vargas-Salgado \\ maria.vargas@uacj.mx
}

\section{Karla Gabriela Gómez-Bull \\ karla.gomez@uacj.mx}

\section{Manuel Alberto Rodríguez-Esparza \\ mardgz@uacj.mx}

Universidad Autónoma de Ciudad Juárez

Chihuahua-México

\section{RESUMEN}

Es común que las organizaciones se enfrentan a cambios desafiantes, ante esto el comportamiento ciudadano de las personas puede afectar o contribuir en el éxito de la firma. Es imperante que los administradores pongan atención a ello y consideren que un entorno apropiado contribuirá considerablemente en la participación y comportamiento de las personas. El objetivo fue analizar si los comportamientos de ciudadanía organizacional influyen sobre la cultura adhocrática, el compromiso organizacional y la satisfacción laboral de los empleados que laboran en empresas de servicio de la frontera norte y realizan un trabajo de forma directa con el cliente. El estudio fue de tipo no experimental, transeccional, correlacional-causal y con un enfoque cuantitativo, se consideró una muestra por conveniencia de 180 empleados. Los datos se obtuvieron por medio de una encuesta administrada directamente a cada participante. Los datos obtenidos fueron examinados mediante mínimos cuadrados parcializados [PLS]. El modelo se corrió con el software SmartPLS. Los resultados demuestran que las relaciones planteadas son estadísticamente significativas: Los CCO impactan de forma directa a la 
cultura adhocrática, compromiso organizacional y satisfacción laboral, y también de forma indirecta con la satisfacción laboral a través de la mediación de la cultura adhocráticas y compromiso organizacional.

Palabras clave: Comportamientos de ciudadanía organizacional; culturas adhocráticas; compromiso organizacional; satisfacción laboral. 


\title{
Organizational citizenship behaviors as antecedent of culture, commitment and job satisfaction in service companies
}

\begin{abstract}
It is common for organizations to face challenging changes, before this the citizen behavior of people can affect or contribute to the success of the firm. It is imperative that managers pay attention to this and consider that an appropriate environment will contribute significantly to people's participation and behavior. The objective was to analyze whether organizational citizenship behaviors influence adhocratic culture, organizational commitment and job satisfaction of employees who work in service companies on the northern border and perform work directly with the customer. The study was non-experimental, transactional, correlational, and quantitative, a convenience sample of 180 employees was considered. The data were obtained through a survey administered directly to each participant. The data obtained were examined using partial least squares [PLS]. The model was run with SmartPLS software. The results show that the relationships raised are statistically significant: CCOs directly impact adhocratic culture, organizational commitment, and job satisfaction, and indirectly with job satisfaction through the mediation of adhocratic culture and organizational commitment.
\end{abstract}

Keywords: organizational citizenship behaviors; adhocratic cultures; organizational commitment; job satisfaction.

Artículo recibido: 30 noviembre. 2021 Aceptado para publicación: 29 diciembre 2021

Correspondencia: maria.vargas@uacj.mx Conflictos de Interés: Ninguna que declarar 


\section{INTRODUCCIÓN}

Desde siempre, el recurso humano ha sido vital para el funcionamiento de las organizaciones, por ello, se deben gestionar apropiadamente para lograr el equilibrio entre la empresa y los empleados, ya que a través de las competencias que éstos poseen, llevan a la empresa a posicionarse en un mercado global y competitivo (Mappamiring et al., 2020). Frecuentemente en el entorno laboral se presentan desafíos y amenazas, ante esta situación, las firmas se ven obligadas a ofrecer entornos atractivos, con la finalidad de lograr el éxito empresarial (Castillo, 2012; Vieira, 2014).

Generalmente los empleados cuentan con diferentes habilidades y aptitudes. Las personas, cotidianamente viven en entornos cambiantes y complicados, lo que provoca que éstos muestren diversos comportamientos que inciden en los resultados de la firma (Segredo, 2013). En particular, los comportamientos de ciudadanía organizacional [CCO] son relevantes en el contexto laboral, ya que cuando el empleado muestra un comportamiento positivo y decide de forma voluntaria apoyar a sus compañeros y a la organización, éstos logran los objetivos tanto grupales como organizacionales (Wahyu, 2013), es así que los administradores deben de considerar que un entorno laboral apropiado aumenta la participación de los empleados en este tipo de comportamientos (Amini et al., 2018).

Por otra parte, las empresas se preocupan por brindar oportunidad de crecimiento a los empleados, es importante que este crecimiento esté ligado a las demandas laborales de la firma, es decir, a la cultura organizacional de la compañía (Ayala, 2014). Las firmas tienen su propia personalidad en lo que concierne a principios, convicciones, costumbres, valores y forma de operar (Minsal \& Pérez, 2007). Es así como estos aspectos se sujetan en la manera de proceder de los trabajadores dentro de la empresa, y que además se vinculan fuertemente con la historia y filosofía organizacional (Tinoco et al., 2014).

Además, el compromiso es un constructo relevante en el contexto empresarial, ya que a través de éste las compañías fortalecen los lazos laborales con los empleados, es decir, los trabajadores motivados se sentirán parte de esta (Patrón-Cortés \& Canul, 2017; Peña et al., 2016). Por lo tanto, implica la aceptación y entusiasmo de las personas para ser parte de la organización con el fin de participar en el éxito de esta (Novitasari et al., 2020). Es de resaltar que la satisfacción de los empleados en el entorno empresarial es de gran relevancia, ya que es a través de ésta que las personas exteriorizan su agrado con la 
empresa y con lo que en ella realizan, impactando así considerablemente en el buen desempeño y en el crecimiento de la organización (Novitasari et al., 2020). Además, la presencia de diversos factores relacionados con el trabajo impacta en la actitud y disponibilidad de la persona, es decir, muestran una actitud positiva o negativa ante lo que se presenta en el entorno (Amini et al., 2018).

En la frontera norte lo que más prevalece es la industria manufacturera de exportación, la cual es una de las principales generadoras de empleo y economía de la región, sin embargo, la presencia de empresas dedicadas al servicio, también contribuyen considerablemente a la economía. En los últimos años, la industria cinematográfica ha crecido considerablemente y ha generado un gran número de empleos (Ortiz, 2018). Además, el crecimiento de las cadenas de supermercados ha creado múltiples opciones de consumo y un importante crecimiento, posicionando a ese tipo de empresas como grandes contribuyentes de la economía de la zona (Casado, 2018). Por ello de acuerdo con lo anterior, el objetivo fue analizar si los comportamientos de ciudadanía organizacional influyen sobre la cultura adhocrática, el compromiso organizacional y la satisfacción laboral de los empleados que laboran en empresas de servicio de la frontera norte y que realizan un trabajo de forma directa con el cliente.

\section{REVISIÓN DE LITERATURA}

\subsection{Comportamiento de ciudadanía organizacional}

En el contexto organizacional, los dirigentes deben poner atención en lo que sucede en el lugar de trabajo, y conocer de cerca los comportamientos, actitudes y sensibilidad de los empleados, ya que esto puede impactar en el desempeño, permitiendo a las empresas lograr ventajas competitivas operacionales y sostenibles (Singh \& Singh, 2019).

El comportamiento de los empleados y las conductas voluntarias o involuntarias en el lugar de trabajo es lo que se conoce como un CCO, este concepto se caracteriza por ser complejo y que emerge como un aspecto de gran importancia para el entendimiento del comportamiento humano en el lugar de trabajo (Organ, 1997). Organ (1988), define el CCO como un actuar en el comportamiento circunstancial de las personas y que es parte importante en la operación de la organización. El comportamiento como tal no es un requisito exigible en el rol de trabajo o en la descripción de puesto, sino que es una cuestión de decisión personal. En cada situación laboral se encuentran restricciones que 
pueden limitar la capacidad de un individuo en su desempeño con respecto al trato recibido por parte del superior (Coyle-Shapiro, 2002; Lambert, 2000).

Cuando las organizaciones logran que los empleados tengan comportamientos y conductas de ayuda voluntaria con sus compañeros o con la organización, los empleados manifestaran una tendencia de obligación hacia la organización, generando una cultura de comportamientos de ciudadanía organizacional (Montalbán et al., 2014). Además, una cultura innovadora propicia un entorno creativo, flexible y desafiante (Yu, 2017).

Es de destacar que los CCO en los sujetos fortalece la satisfacción de estos en el contexto organizacional, además de puntualizar que las emociones de los trabajadores son un gran aliado en el compromiso de las personas en el lugar de trabajo (Lavy \& Littman-Ovadia, 2016; Singh \& Singh, 2019). En un estudio de maestros universitarios se evidencia una relación entre los comportamientos y el compromiso de los empleados, es decir a partir de la actitud que estos muestran al realizar el trabajo aumenta el compromiso para con la organización (Ahad et al., 2021). Por lo anterior se generan las siguientes hipótesis:

- H1: Los CCO impactan positiva y significativamente en la cultura adhocrática

- H2: Los CCO impactan positiva y significativamente sobre la satisfacción laboral

- H3: Los CCO impactan positiva y significativamente sobre el compromiso organizacional

\subsection{Cultura organizacional}

En el entorno de trabajo, las relaciones laborales y la manera de realizar las actividades engloban una cultura organizacional, la cual es única e irrepetible. Es así como la cultura se considera una virtud y fortaleza que la organización tiene y es lo que la hace distinta de sus competidores (Steckerl, 2011). Ésta puede definir la manera en la que se desarrollan las relaciones humanas y puede causar un efecto positivo o negativo dentro de la empresa, sirviendo como guía de conducta de los trabajadores dentro de la firma, con el propósito de cumplir los objetivos de esta (Dimitrova \& Marín, 2006). Al mismo tiempo ésta puede modificarse de acuerdo con las necesidades de las personas y la empresa (Gastélum, 2000).

Schein (1986), menciona que la cultura se caracteriza por comportamientos que se originan dentro del grupo de trabajo y éstos contribuyen en generar identidad de los empleados para con la organización, esta cultura se da entre los empleados y los nuevos integrantes. Al respecto Cameron y Quinn (2011), mencionan que las áreas funcionales, 
departamentos y grupos de trabajo, pueden reflejar su propia cultura. Además, mencionan que existen cuatro tipos de cultura: clan, jerárquica, de mercado y adhocrática. En particular la cultura adhocrática se enfoca en la innovación y espíritu empresarial, asimismo los atributos como la flexibilidad, adaptabilidad y creatividad fomentan una actitud de riesgo entre los empleados (Cameron \& Quinn, 2011; Engelen et al., 2014). Una buena cultura organizacional creará compromiso entre los integrantes de la organización, ayudará a lograr las metas y facilitará el espacio compartido entre los empleados (Polychroniou \& Trivellas, 2018; Yanti \& Dahlan, 2017). Esto implica que al existir una cultura adecuada impactara considerablemente en el compromiso de las personas (Yusuf, 2020). La investigación de Lok y Crawford (2004), revelan una relación directa entre la cultura y el compromiso organizacional. Por lo anterior se postula que:

- H4: La cultura adhocrática influye positiva y significativamente sobre el compromiso organizacional

\subsection{Compromiso organizacional}

En el contexto laboral el compromiso se da cuando los trabajadores perciben que vale la pena permanecer en la empresa, las personas que están altamente comprometidos con la firma manifiestan un buen desempeño, iniciativa y colaboración, no obstante, cuando no están comprometidos reflejarán todo lo contrario (Sánchez \& Franco, 2019). Mowday et al. (1979), refieren que el compromiso involucra la colaboración que un empleado tiene para con la empresa y la intención que de estar en ella. Para Robbins y Judge (2009), es la identificación de los sujetos con las metas de la organización. Mientras que para Meyer et al. (1993), es el estado psicológico que caracteriza la relación laboral entre el empleado y la empresa, la cual implica la decisión de permanecer en la organización.

Meyer y Allen (1991) mencionan que el compromiso organizacional integra tres dimensiones [afectivo, de continuidad y normativo] que engloban el constructo. La primera de ellas hace referencia a la parte emocional del empleado, es decir, el empleado continua en la empresa porque así lo desea; la segunda está ligada a la parte de los beneficios que el trabajador percibe por parte de la empresa, como beneficios monetarios, y abandonar implicaría perder todo; finalmente el tercero consiste en la gratitud que el trabajador le tiene a la empresa por la oportunidad que le han dado. Cabe destacar que, el compromiso de los empleados dependerá de algunos factores, entre ellos, el puesto que desempeña, el salario que percibe, la jornada laboral, entre otros. No obstante, se entiende 
que los empleados pueden tener ciertos privilegios por parte de la empresa, lo cual se traduce en compromiso de permanecer o por el contrario de abandonar la organización (Barraza, 2008).

Estudios previos revelan que el compromiso está relacionado con la satisfacción de los empleados, es así como, entre mayor compromiso, mayor satisfacción por parte de los trabajadores (Rahmawati \& Tobing, 2019). Por otra parte, la estabilidad laboral aumenta el nivel de satisfacción y compromiso de las personas (AlKahtani et al., 2021). Además, cuando las empresas promueven el compromiso, la estancia de los trabajadores se prolonga dado que se encuentran contentos con su trabajo (Loan, 2020). Por lo anterior

\section{se postula que:}

- H5: El compromiso organizacional impacta positiva y significativamente sobre la satisfacción laboral

\subsection{Satisfacción laboral}

La satisfacción en el trabajo representa la medida en que se cumplen las expectativas de los individuos y como éstas coinciden con los resultados y generan una sensación al respecto en el entorno laboral. Por ello, el entorno y las relaciones laborales son un factor esencial para que las personas estén satisfechas o insatisfechas en el lugar de trabajo (Paais \& Pattiruhu, 2020).

Spector (1985), relaciona la satisfacción laboral directamente con la manera de como las personas se sienten acerca de su trabajo y otros aspectos, lo que permite obtener una aproximación de hasta qué punto las personas se encentran satisfechas o descontentas con su trabajo. Por otra parte, se considera como "una representación de la combinación de sentimientos positivos o negativos que los empleados tienen hacia sus trabajos" (Aziri, 2011, p. 78).

La teoría de los dos factores de Herzberg es la más citada en la literatura, la idea principal es que los empleados dentro del ambiente laboral se encuentran bajo la influencia de factores que causan la satisfacción laboral a través de motivadores [logro, reconocimiento, crecimiento] y factores de higiene [supervisión, condiciones de trabajo, salario y seguridad en el trabajo] (Herzberg 1976). Es importante destacar que estos factores pueden causar insatisfacción laboral, por lo tanto, la satisfacción y la insatisfacción no pueden ser abordados como elementos excluyentes entre sí (Aziri, 2011). Es así como la satisfacción laboral se encuentra bajo una interminable serie de 
factores que influyen en la percepción de ésta y su entendimiento en el área organizacional. El estudio de la variable de manera puntual y óptima puede generar resultados positivos en el cumplimiento de objetivos y desempeño de la organización.

\section{METODOLOGÍA}

El estudio fue de tipo no experimental, transeccional, correlacional-causal y con un enfoque cuantitativo, se consideró una muestra no probabilística por conveniencia de 180 participantes, los sujetos de estudio fueron empleados que laboran en empresas de servicio de la frontera norte y que desarrollan su trabajo de forma directa con el cliente. Para la recolección de datos se empleó un cuestionario administrado directamente a cada uno de los sujetos en su lugar de trabajo, los datos fueron recabados durante los meses de febrero y marzo de 2020.

Para medir cada uno de los constructos de estudio, se adaptaron escalas previamente validadas (tabla1), para ello se utilizó una escala Likert de 5 puntos donde nunca=1 y siempre $=5$, y en algunos casos desde muy en desacuerdo hasta muy de acuerdo. Una vez recabados los datos, se procedió a obtener los estadísticos descriptivos e inferenciales correspondientes. Los estadísticos descriptivos se examinaron con la herramienta Statistical Package for the Social Sciences [SPSS] mientras que la inferencial se analizó mediante el software SmartPLS 3.0.

Tabla 1. Operacionalización de constructos

\begin{tabular}{llc}
\hline Constructo & Adaptación & Ítems \\
\hline CCO & Lee and Allen (2002) & 16 \\
Cultura adhocrática & Cameron and Quinn (2011) & 9 \\
Compromiso organizacional & Allen and Meyer (1990) & 23 \\
Satisfacción laboral & Meliá and Peiró (1989) & 21 \\
\hline
\end{tabular}

Fuente: elaboración a partir de Allen \& Meyer, 1990; Cameron \& Quinn, 2011; Lee \& Allen, 2002; Meliá \& Peiró, 1989.

\section{RESULTADOS Y DISCUSIÓN}

En la tabla 2 se presentan las características sociodemográficas de los participantes, se aprecia que el 58.9\% son mujeres, el 70\% de la muestra nació entre 1981 y 2000, es decir tienen entre 21 y 40 años. Con respecto a la antigüedad en el lugar de trabajo, el $46.7 \%$ de los participantes tiene entre 1 a 5 años trabajando en la organización. En la escolaridad, se observa que el $31.7 \%$ cuenta con estudios de bachillerato y el $15 \%$ con preparación académica menor a bachillerato. Finalmente, la muestra estuvo conformada por trabajadores pertenecientes a diferentes empresas de servicios, destacando la 
participación de las empresas de entretenimiento, donde el $42.8 \%$ de los participantes de la muestra labora en éstas.

Tabla 2. Características sociodemográficas $n=180$

\begin{tabular}{llrlrr}
\multicolumn{1}{c}{ Categoría } & Personas & \% & \multicolumn{1}{c}{ Categoría } & Personas & \% \\
\hline Sexo & & & Escolaridad & & \\
Hombre & 74 & 41.1 & Menor a bachillerato & 27 & 15.0 \\
Mujer & 106 & 58.9 & Bachillerato & 57 & 31.7 \\
Año de nacimiento & & & Carrera técnica & 19 & 10.6 \\
Entre 1960 y 1980 & 20 & 11.1 & Carrera profesional trunca & 43 & 23.9 \\
Entre 1981 y 2000 & 126 & 70.0 & Carrera profesional terminada & 30 & 16.7 \\
Del 2000 en adelante & 34 & 18.9 & Posgrado & 4 & 2.2 \\
Antigüedad & & & & & \\
Menor a 1 año & 67 & 37.2 & Empresa & 77 & 42.8 \\
De 1 año a 5 años & 84 & 46.7 & Entretenimiento & 45 & 25.0 \\
De 6 años a 10 años & 22 & 12.2 & Supermercados & 28 & 15.6 \\
De 11 años a 15 años & 2 & 1.1 & Gaseras & 30 & 16.6 \\
De 16 años a 20 años & 4 & 4.0 & Servicio general & & \\
Mayor a 20 años & 1 & 0.6 & & & \\
\hline
\end{tabular}

Fuente: Elaboración propia

En la tabla 3 se exhiben las cargas y valor t de cada uno de los indicadores que integran los constructos del modelo. La carga factorial es un indicador que mide la fiabilidad de la varianza que el indicador comparte con el constructo, se recomienda que el punto de corte sea superior a 0.70 (Fornell \& Larcker, 1981), al respecto Chin (1998), señala que puede haber cargas de 0.50 y estas pueden ser aceptadas siempre y cuando en el constructo se exhiba indicadores con cargas altas. En este caso, la mayoría de los indicadores de las cargas son mayores al valor aceptable a excepción de [CCOP1, CCOP3, CCOP7], [CO2, CO12, CO14, CO20] y [SA1, SA13, SA14, SA16, SA21, SA22] los cuales presentan valores inferiores al punto de corte. Con respecto a los valores t el valor mínimo es 7.561 y máximo 33.016, ante esto se puede afirmar que todos los valores son superiores a 3.29 por lo cual son estadísticamente significativos.

También se muestran los indicadores alfa de Cronbach, análisis de fiabilidad compuesta [IFC] y varianza extraída [AVE]. Usualmente la fiabilidad se valora a través del alfa de Cronbach. Sin embargo, Hair, Anderson, Tatham, y Black (1999), refieren que el IFC es un mejor indicador de consistencia interna que valora la correlación entre cada uno de los ítems que conforman el constructo, para ambos indicadores se recomienda un punto de corte de 0.70 (Fornell \& Bookstein, 1982). En ambos indicadores se cumple con el punto de corte requerido, alfa de Cronbach: CCO [0.894], cultura adhocrática [0.929], 
compromiso organizacional [0.924] y satisfacción laboral [0.924]; en el caso del IFE: CCO [0.914], cultura adhocrática [0.941], compromiso organizacional [0.934] y satisfacción laboral [0.934]. Con respecto al índice AVE, esta estima la varianza que representa cada indicador, en este caso se deberá presentar valores superiores a 0.50 (Chin, 1998; Gefen \& Straub, 2005). En este estudio todos los indicadores cumplen con el punto de corte CCO [0.522], cultura adhocrática [0.639], compromiso organizacional [0.525] y satisfacción laboral [0.503].

Tabla 3. Indicadores del modelo de medida

\begin{tabular}{|c|c|c|c|c|c|c|c|}
\hline \multicolumn{2}{|c|}{$\mathrm{CCO}$} & \multicolumn{2}{|c|}{$\begin{array}{c}\text { Cultura } \\
\text { adhocrática }\end{array}$} & \multicolumn{2}{|c|}{$\begin{array}{c}\text { Compromiso } \\
\text { organizacional }\end{array}$} & \multicolumn{2}{|c|}{ Satisfacción laboral } \\
\hline CCOE2 & $\begin{array}{l}\mathrm{c}=0.706 \\
{[\mathrm{t}=24.469]}\end{array}$ & CA1 & $\begin{array}{l}\mathrm{c}=0.807 \\
{[\mathrm{t}=25.495]}\end{array}$ & $\mathrm{CO} 1$ & $\begin{array}{l}\mathrm{c}=0.752 \\
{[\mathrm{t}=18.879]}\end{array}$ & SA1 & $\begin{array}{l}\mathrm{c}=0.655 \\
{[\mathrm{t}=11.641]}\end{array}$ \\
\hline CCOE3 & $\begin{array}{l}\mathrm{c}=0.790 \\
{[\mathrm{t}=26.573]}\end{array}$ & CA2 & $\begin{array}{l}\mathrm{c}=0.822 \\
{[\mathrm{t}=32.796]}\end{array}$ & $\mathrm{CO} 10$ & $\begin{array}{l}\mathrm{c}=0.638 \\
{[\mathrm{t}=12.073]}\end{array}$ & SA11 & $\begin{array}{l}c=0.725 \\
{[t=16.528]}\end{array}$ \\
\hline CCOE4 & $\begin{array}{l}\mathrm{c}=0.810 \\
{[\mathrm{t}=32.881]}\end{array}$ & CA3 & $\begin{array}{l}\mathrm{c}=0.814 \\
{[\mathrm{t}=27.952]}\end{array}$ & $\mathrm{CO} 12$ & $\begin{array}{l}c=0.628 \\
{[t=9.757]}\end{array}$ & SA12 & $\begin{array}{l}\mathrm{c}=0.780 \\
{[\mathrm{t}=23.553]}\end{array}$ \\
\hline CCOE5 & $\begin{array}{l}\mathrm{c}=0.750 \\
{[\mathrm{t}=19.182]}\end{array}$ & CA4 & $\begin{array}{l}\mathrm{c}=0.851 \\
{[\mathrm{t}=32.362]}\end{array}$ & $\mathrm{CO} 14$ & $\begin{array}{l}c=0.625 \\
{[t=10.756]}\end{array}$ & SA13 & $\begin{array}{l}\mathrm{c}=0.632 \\
{[\mathrm{t}=11.532]}\end{array}$ \\
\hline CCOE6 & $\begin{array}{l}\mathrm{c}=0.762 \\
{[\mathrm{t}=22.006]}\end{array}$ & CA5 & $\begin{array}{l}\mathrm{c}=0.781 \\
{[\mathrm{t}=20.760]}\end{array}$ & $\mathrm{CO} 2$ & $\begin{array}{l}c=0.773 \\
{[t=23.606]}\end{array}$ & SA14 & $\begin{array}{l}\mathrm{c}=0.677 \\
{[\mathrm{t}=12.189]}\end{array}$ \\
\hline CCOE7 & $\begin{array}{l}\mathrm{c}=0.817 \\
\mathrm{t}=29.129]\end{array}$ & CA6 & $\begin{array}{l}\mathrm{c}=0.796 \\
{[\mathrm{t}=21.098]}\end{array}$ & $\mathrm{CO} 20$ & $\begin{array}{l}c=0.621 \\
{[t=11.357]}\end{array}$ & SA16 & $\begin{array}{l}\mathrm{c}=0.654 \\
{[\mathrm{t}=12.688]}\end{array}$ \\
\hline CCOE8 & $\begin{array}{l}\mathrm{c}=0.847 \\
{[\mathrm{t}=33.016]}\end{array}$ & CA7 & $\begin{array}{l}\mathrm{c}=0.801 \\
{[\mathrm{t}=25.000]}\end{array}$ & $\mathrm{CO} 22$ & $\begin{array}{l}c=0.738 \\
{[t=17.993]}\end{array}$ & SA17 & $\begin{array}{l}\mathrm{c}=0.704 \\
{[\mathrm{t}=16.010]}\end{array}$ \\
\hline CCOP1 & $\begin{array}{l}\mathrm{c}=0.541 \\
{[\mathrm{t}=7.561]}\end{array}$ & CA8 & $\begin{array}{l}\mathrm{c}=0.777 \\
{[\mathrm{t}=18.586]}\end{array}$ & $\mathrm{CO} 23$ & $\begin{array}{l}\mathrm{c}=0.725 \\
{[\mathrm{t}=17.009]}\end{array}$ & SA18 & $\begin{array}{l}\mathrm{c}=0.738 \\
{[\mathrm{t}=16.308]}\end{array}$ \\
\hline CCOP3 & $\begin{array}{l}\mathrm{c}=0.587 \\
{[\mathrm{t}=9.695]}\end{array}$ & CA9 & $\begin{array}{l}\mathrm{c}=0.742 \\
{[\mathrm{t}=16.024]}\end{array}$ & $\mathrm{CO} 3$ & $\begin{array}{l}c=0.773 \\
{[t=22.270]}\end{array}$ & SA19 & $\begin{array}{l}c=0.711 \\
{[t=12.653]}\end{array}$ \\
\hline CCOP7 & $\begin{array}{l}\mathrm{c}=0.525 \\
{[\mathrm{t}=8.817]}\end{array}$ & & & $\mathrm{CO} 5$ & $\begin{array}{l}c=0.735 \\
{[t=16.973]}\end{array}$ & SA2 & $\begin{array}{l}\mathrm{c}=0.753 \\
{[\mathrm{t}=16.278]}\end{array}$ \\
\hline & & & & CO6 & $\begin{array}{l}\mathrm{c}=0.826 \\
{[\mathrm{t}=26.964]}\end{array}$ & SA20 & $\begin{array}{l}\mathrm{c}=0.723 \\
{[\mathrm{t}=14.164]}\end{array}$ \\
\hline & & & & $\mathrm{CO} 7$ & $\begin{array}{l}\mathrm{c}=0.766 \\
{[\mathrm{t}=23.869]}\end{array}$ & SA21 & $\begin{array}{l}\mathrm{c}=0.689 \\
\mathrm{t}=10.980]\end{array}$ \\
\hline & & & & $\mathrm{CO} 8$ & $\begin{array}{l}\mathrm{c}=0.782 \\
{[\mathrm{t}=18.843]}\end{array}$ & SA22 & $\begin{array}{l}\mathrm{c}=0.686 \\
\mathrm{t}=14.475]\end{array}$ \\
\hline & & & & & & SA23 & $\begin{array}{l}\mathrm{c}=0.786 \\
{[\mathrm{t}=25.216]}\end{array}$ \\
\hline $\mathbf{A C}$ & 0.894 & 0.929 & & 0.924 & & 0.924 & \\
\hline IFC & 0.914 & 0.941 & & 0.934 & & 0.934 & \\
\hline AVE & 0.522 & 0.639 & & 0.525 & & 0.503 & \\
\hline
\end{tabular}

$\mathrm{c}=$ carga, $\mathrm{t}=$ valores $\mathrm{t} . \mathrm{AC}=\mathrm{Alfa}$ de Cronbach, $\mathrm{IFC}=$ Índice de fiabilidad compuesta, AVE=análisis de varianza extraída. *valor t mayor a 1.96 para una prueba de dos colas, *** valor $\mathrm{t}$ mayor a 3.291 para una prueba de dos colas. Fuente: Elaboración a partir de SmartPLS 3 
La validez discriminante se presenta a partir de los indicadores AVE y HeterotraitMonotrait Ratio [HTMT] mismas que se presetan en la tabla 4. En el caso 1 [Criterio Fornell-Larcker] se dice que cuando la raíz cuadrada de la varianza extraída es superior que la compartida, existe validez discriminante (Fornell \& Larcker, 1981). En todos los casos el indicador AVE es superior que las correlaciones, por lo tanto, existe validez discriminante. En el caso 2 [Heterotrait-Monotrait Ratio, HTMT] valora las correlaciones entre los indicadores que evaluan distintos constructos, ésta se puede estimar a traves de criterio o como prueba estadística (Henseler et al., 2015). En este estudio se estima como criterio y se eligió 0.800 como punto de corte. Se aprecia que los ratios cumplen con el indicador, por lo cual tambien existe validez discriminate.

Tabla 4. Validez discriminante

\begin{tabular}{|c|c|c|c|c|}
\hline Constructo & $\mathrm{CCO}$ & $\begin{array}{c}\text { Compromiso } \\
\text { organizacional }\end{array}$ & $\begin{array}{c}\text { Cultura } \\
\text { adhocrática }\end{array}$ & $\begin{array}{c}\text { Satisfacción } \\
\text { laboral }\end{array}$ \\
\hline \multicolumn{5}{|c|}{ Caso 1. Criterio Fornell-Larcker } \\
\hline $\mathrm{CCO}$ & 0.723 & - & - & - \\
\hline $\begin{array}{l}\text { Compromiso } \\
\text { organizacional }\end{array}$ & 0.721 & 0.725 & - & - \\
\hline Cultura adhocrática & 0.659 & 0.723 & 0.800 & - \\
\hline Satisfacción laboral & 0.589 & 0.635 & 0.581 & 0.709 \\
\hline \multicolumn{5}{|c|}{ Caso 2. Heterotrait-Monotrait Ratio [HTMT] } \\
\hline $\mathrm{CCO}$ & - & - & - & - \\
\hline $\begin{array}{l}\text { Compromiso } \\
\text { organizacional }\end{array}$ & 0.774 & - & - & - \\
\hline Cultura adhocrática & 0.712 & 0.827 & - & - \\
\hline Satisfacción laboral & 0.627 & 0.653 & 0.605 & - \\
\hline
\end{tabular}

Fuente: Elaboración a partir de SmartPLS

El modelo estructural se muestra en la figura 1, en el cual se observan los paths y valores $\mathrm{r}^{2}$. En el caso de los 5 paths planteados todos cumplen con el criterio admisible de 0.20: CCO - cultura [0.659], CCO - satisfacción laboral [0.269], CCO - compromiso organizacional [0.391], cultura - compromiso [0.515], compromiso - satisfacción laboral [0.438]. Con respecto al valor $\mathrm{r}^{2}$, este coeficiente permite valorar en qué medida las variables endógenas son explicadas a partir de la predictoras (Chin, 1998). En este caso de forma moderada los CCO explican tanto la cultura adhocrática $\left[\mathrm{r}^{2}=0.435\right]$ como la satisfacción laboral a partir de la mediación del compromiso organizacional $\left[\mathrm{r}^{2}=0.436\right]$ y 
de forma sustancial los CCO explican el compromiso organizacional a partir de la mediación de la cultura adhocrática $\left[\mathrm{r}^{2}=0.684\right]$.

Con respecto a las 5 hipótesis planteadas ninguna de ellas se rechaza, se evidencia que: Los $\mathrm{CCO}\left[\mathrm{H} 2, \mathrm{p}=0.028^{*}\right]$ y el compromiso organizacional $\left[\mathrm{H} 5, \mathrm{p}=0.000^{* * *}\right]$ impactan positiva y significativamente en la satisfacción laboral, los $\mathrm{CCO}\left[\mathrm{H} 3, \mathrm{p}=0.000^{* * *}\right]$ y la cultura adhocrática $\left[\mathrm{H} 4, \mathrm{p}=0.000^{* * *}\right]$ inciden positiva y significativamente sobre el compromiso organizacional, finalmente los $\mathrm{CCO}$ impactan positiva y significativamente en la cultura adhocrática $\left[\mathrm{H} 1, \mathrm{p}=0.000^{* * *}\right]$. En 4 de las 5 hipótesis se aprecian valores $\mathrm{t}$ superiores a 3.291, por lo cual se consideran fiables a un nivel de confianza del 99.9\%, la excepción se encuentra en $\mathrm{H} 2$ la cual es fiable a un nivel de confianza del 95\%.

Figura 1. Modelo estructural

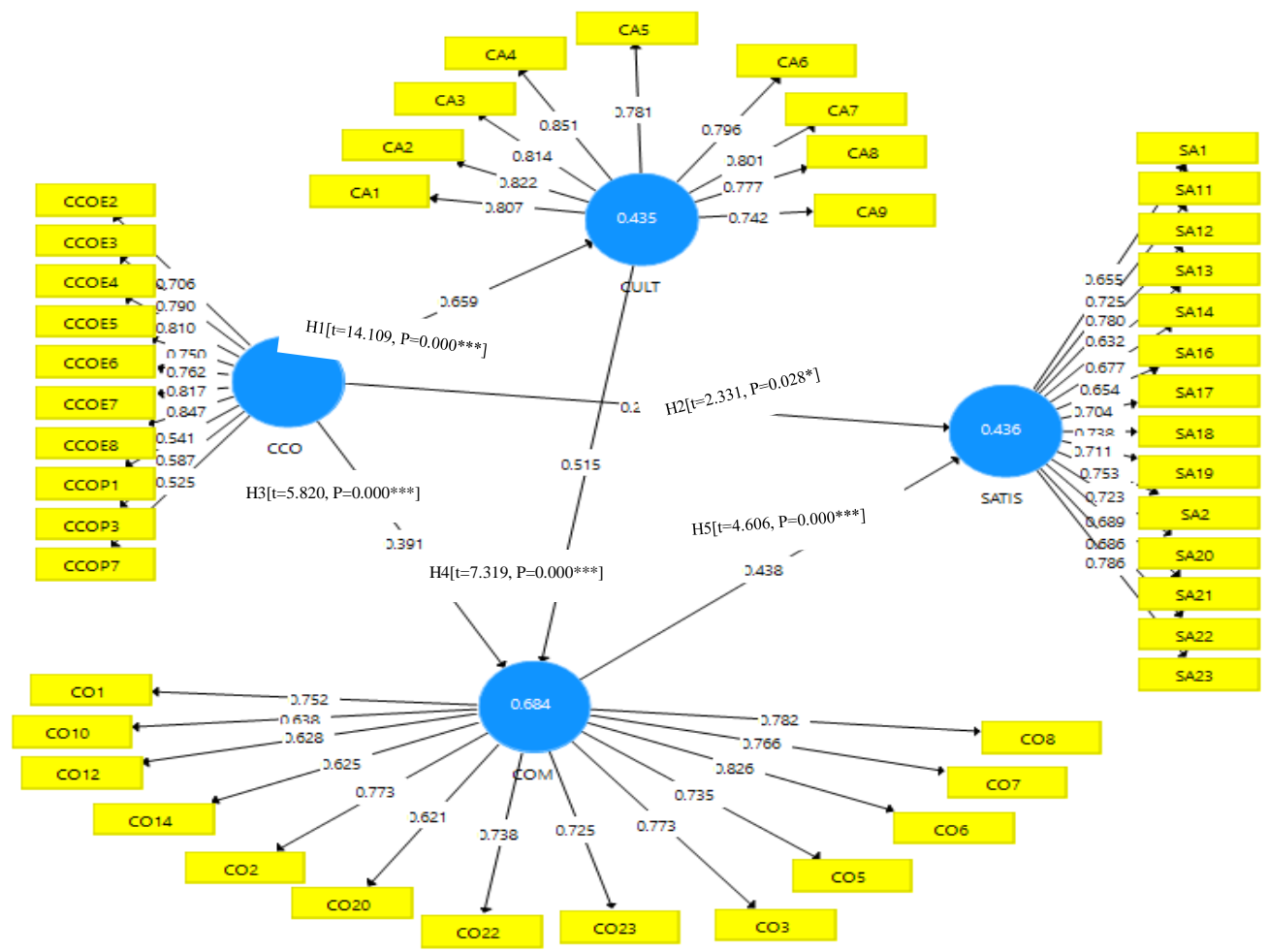

*valor t mayor a 1.96 para una prueba de dos colas, con un Alpha de 0.05

*** valor t mayor a 3.291 para una prueba de dos colas, con un Alpha de 0.001

Fuente: Elaboración a partir de SmartPLS 3

Los resultados puntualizan que todas las relaciones planteadas son estadísticamente significativas, los CCO inciden de forma directa con la cultura adhocrática, el compromiso organizacional y la satisfacción laboral y de forma indirecta a través de la 
mediación de la cultura adhocrática y compromiso organizacional impacta en la satisfacción laboral.

En el contexto de las empresas de servicio los CCO impactan en la cultura adhocrática, en la satisfacción laboral y el compromiso organizacional. En este tipo de empresas las personas manifiestan compartir herramientas personales para ayudar a sus compañeros en el trabajo, se ayudan entre sí, es decir comparten algunas responsabilidades cuando consideran que es necesario, además hacen todo lo posible para que los nuevos integrantes se sientan bienvenidos a la empresa, esto coincide con lo planteado por Montalbán et al. (2014), quienes refieren que cuando los trabajadores tienen comportamientos y conductas de ciudadanía organizacional ayudan por iniciativa propia a sus compañeros de trabajo. Asimismo, en este contexto se destaca la cultura adhocrática que prevalece en el lugar de trabajo, ya que la innovación, la iniciativa y la búsqueda de oportunidades, propician a realizar el trabajo de forma desafiante. Ante esto, es imperante resaltar la relevancia de una cultura creativa, flexible y retadora (Yu, 2017).

Por otra parte, en el centro de trabajo se propicia un ambiente de negociación en relación con las actividades a desempeñar ya que se da la oportunidad a los empleados de realizar lo que les gusta, en este caso tener un trato directo con el cliente, donde las emociones son un gran aliado para generar CCO. Los resultados concuerdan con estudios previos, mismos que resaltan que las personas que manifiestan CCO se sienten satisfechos con las responsabilidades asignadas en el centro de trabajo (Lavy \& Littman-Ovadia, 2016; Singh \& Singh, 2019).

Es importante destacar que los empleados con $\mathrm{CCO}$ fortalecen el compromiso que éstos tienen para con la empresa (Ahad et al., 2021). En este estudio las personas manifiestan un compromiso afectivo para con la empresa, es decir permanecen en la organización porque les agrada el trabajo que realizan, además les gusta lo que hacen, por ello disfrutan hablar acerca de la organización con los clientes. Lo anterior coincide con trabajos previos (AlKahtani et al., 2021; Loan, 2020; Rahmawati \& Tobing, 2019) quienes refieren que cuando los empleados se encuentran contentos con su trabajo estos permanecen por largo tiempo.

\section{CONCLUSIONES}

El estudio impacta en el contexto organizacional, ya que con sus hallazgos destaca la importancia de que las organizaciones fortalezcan la buena cultura organizacional, en 
donde se promuevan las buenas conductas y comportamientos de los miembros de las firmas, ya que como se evidenció, éstas impactan en el cumplimiento de metas organizacionales.

Con la evidencia proporcionada a través de este estudio, se destaca la necesidad de que las organizaciones pongan atención en las conductas de ciudadanía organizacional, ya que es a través de éstas que se logra aumentar la satisfacción de los empleados, se influye sobre la cultura adhocrática y a su vez incrementa el compromiso de los trabajadores hacia la organización. De forma tal que, el recurso humano se mantenga comprometido y alineado a trabajar encaminado hacia los mismos objetivos planteados por la organización y hacer frente a la alta competitividad con la que se cuenta actualmente.

En este trabajo se utilizó un diseño transversal y una muestra no probabilística, por lo cual los resultados no pueden generalizarse. Es por lo que se considera conveniente, que en trabajos futuros se consideren estudios longitudinales y muestras probabilísticas, además de incluir otros contextos de estudio tales como restaurantes, hoteles, aerolíneas, salón de eventos, belleza, entre otros. También se piensa que se deberían de abordar otras variables como antecedentes y consecuentes de los CCO.

\section{REFERENCIAS BIBLIOGRAFICAS}

Ahad, R., Mustafa, M. Z., Mohamad, S., Abdullah, N. H. S., \& Nordin, M. N. (2021). Work Attitude, Organizational Commitment and Emotional Intelligence of Malaysian Vocational College Teachers. Journal of Technical Education and Training, 13(1), 15-21. https://doi.org/10.30880/jtet.2021.13.01.002

AlKahtani, N., Iqbal, S., Sohail, M., Sheraz, F., Jahan, S., Anwar, B., \& Haider, S. (2021). Impact of employee empowerment on organizational commitment through job satisfaction in four and five stars hotel industry. Management Science Letters, 11(3), 813-822. https://doi.org/10.5267/j.ms1.2020.10.022

Allen, N. J., \& Meyer, J. P. (1990). The measurement and antecedents of affective, continuance and normative commitment to the organization. Journal of occupational psychology, 63(1), 1-18. $\quad$ https://doi.org/10.1111/j.20448325.1990.tb00506.x

Amini, N., Rezaei, T., \& Tavakoli, M. (2018). The study of mediator role of job satisfaction in organizational justice and citizenship behavior relationship in one 
of the Shiraz factories. UCT Journal of Social Sciences and Humanities Research, 6(04), 79-84. https://doi.org/10.24200/jsshr.vol6iss04pp79-84

Ayala, P. (2014). Cultura Organizacional e Innovación. Revista Electrónica Gestión de las Personas y Tecnología, 7(19), 44-55.

Aziri, B. (2011). Job Satisfaction: A Literature Review. Management Research \& Practice, 3(4).

Barraza, A. (2008). Compromiso organizacional de los docentes: un estudio exploratorio.

Avances en supervisión educativa: Revista de la Asociación de Inspectores de Educación de España(8), 20.

Cameron, K. S., \& Quinn, R. E. (2011). Diagnosing and changing organizational culture: Based on the competing values framework: John Wiley \& Sons.

Casado, J. M. C. (2018). Supermercados en México: expansión y espacios de inserción. Estudios Geográficos, 79(284), 167-190.

Castillo, R. D. C. (2012). Desarrollo del capital humano en las organizaciones (Primera ed.). México: RED TERCER MILENIO SC.

Chin, W. (1998). Issues and Opinion on Structural Equation Modeling. MIS Quarterly, 22(1), 7-16.

Coyle-Shapiro, J. A. M. (2002). A psychological contract perspective on organizational citizenship behavior. Journal of Organizational Behavior: The International Journal of Industrial, Occupational and Organizational Psychology and Behavior, 23(8), 927-946. https://doi.org/10.1002/job.173

Dimitrova, S., \& Marín, L. (2006). El concepto de cultura de las organizaciones: Centralidad actual y evolución. Revista internacional de organizaciones, 65-76.

Engelen, A., Flatten, T. C., Thalmann, J., \& Brettel, M. (2014). The Effect of Organizational Culture on Entrepreneurial Orientation: A Comparison between Germany and Thailand. Journal of Small Business Management, 52(4), 732-752. https://doi.org/10.1111/jsbm.12052

Fornell, C., \& Bookstein, F. L. (1982). Two Structural Equation Models: LISREL and PLS Applied to Consumer Exit-Voice Theory. Journal of Marketing Research, 19(4), 440-452. https://doi.org/10.1177/002224378201900406 
Fornell, C., \& Larcker, D. F. (1981). Evaluating Structural Equation Models with Unobservable Variables and Measurement Error. Journal of Marketing Research, 18(1), 39-50. https://doi.org/10.1177/002224378101800104

Gastélum, R. G. (2000). Comunicación y cultura organizacional en empresas chinas y japonesas: Juan Carlos Martínez Coll.

Gefen, D., \& Straub, D. (2005). A Practical Guide to Factorial Validity Using PLS-Graph: Tutorial and Annotated Example. Communications of the Association for Information Systems, 16, 91-109.

Hair, J. F., Anderson, R. F., Tatham, R. L., \& Black, W. C. (1999). Análisis Multivariante (5ta Edición ed.). Madrid: Prentice Hall Iberia.

Henseler, J., Ringle, C. M., \& Sarstedt, M. (2015). A new criterion for assessing discriminant validity in variance-based structural equation modeling. Journal of the Academy of Marketing Science, 43(1), 115-135. https://doi.org/10.1007/s11747-014-0403-8

Lambert, S. J. (2000). Added benefits: The link between work-life benefits and organizational citizenship behavior. Academy of management Journal, 43(5), 801-815.

Lavy, S., \& Littman-Ovadia, H. (2016). My Better Self: Using Strengths at Work and Work Productivity, Organizational Citizenship Behavior, and Satisfaction. Journal of Career Development, 44(2), 95-109. https://doi.org/10.1177/0894845316634056

Lee, K., \& Allen, N. J. (2002). Organizational citizenship behavior and workplace deviance: The role of affect and cognitions. Journal of applied psychology, 87(1), 131. https://doi.org/10.1037//0021-9010.87.1.131

Loan, L. (2020). The influence of organizational commitment on employees' job performance: The mediating role of job satisfaction. Management Science Letters, 10(14), 3307-3312. doi: 10.5267/j.msl.2020.6.007

Lok, P., \& Crawford, J. (2004). The effect of organisational culture and leadership style on job satisfaction and organisational commitment. Journal of Management Development, 23(4), 321-338. https://doi.org/10.1108/02621710410529785

Mappamiring, M., Akob, M., \& Putra, A. H. P. K. (2020). What Millennial Workers Want? Turnover or Intention to Stay in Company. The Journal of Asian Finance, 
Economics, and Business, 7(5), 237-248. doi: https://doi.org/10.13106/JAFEB.2020.VOL7.NO5.237

Meliá, J., \& Peiró, J. (1989). La medida de la satisfacción laboral en contextos organizacionales: El Cuestionario de Satisfacción S20/23. Psicologemas, 5(2), 59-74.

Meyer, J. P., \& Allen, N. J. (1991). A Three-Component Conceptualization of Organizational Commitment. Human Resource Management, 1(1), 61-89. https://doi.org/10.1016/1053-4822(91)90011-Z

Meyer, J. P., Allen, N. J., \& Smith, C. A. (1993). Commitment to organizations and occupations: Extension and test of a three-component conceptualization. Journal of applied psychology, 78(4), 538. https://doi.org/10.1037/0021-9010.78.4.538

Minsal, D., \& Pérez, Y. (2007). Hacia una nueva cultura organizacional: la cultura del conocimiento. Acimed, 16(3), 0-0.

Montalbán, R. L. R., Soria, M. S., \& Lugo, M. M. (2014). Justicia organizacional, engagement en el trabajo y comportamientos de ciudadanía organizacional: una combinación ganadora. Universitas Psychologica, 13(3), 15-28. https://doi.org/10.11144/Javeriana.UPSY13-3.joet

Mowday, R. T., Steers, R. M., \& Porter, L. W. (1979). The Measurement of Organizational Commitment. Journal of Vocational Behavior 14, 224-247. https://doi.org/10.1016/0001-8791(79)90072-1

Novitasari, D., Asbari, M., Wijaya, M. R., \& Yuwono, T. (2020). Effect of Organizational Justice on Organizational Commitment: Mediating Role of Intrinsic and Extrinsic Satisfaction. International Journal of Science and Management Studies (IJSMS), 3(3), 96-112.

Organ, D. W. (1988). Organizational citizenship behavior: The good soldier syndrome: Lexington Books/DC Heath and Com.

Organ, D. W. (1997). Organizational citizenship behavior: It's construct clean-up time. Human performance, 10(2), 85-97.

Ortiz, L. (2018). La industria cinematográfica en México, tan fuerte como la del hierro o el acero, Expansión. Retrieved from https://expansion.mx/vidaarte/2019/06/14/la-industria-cinematografica-en-mexico-tan-fuerte-como-la-delhierro-o-el-acero 
Paais, M., \& Pattiruhu, J. R. (2020). Effect of motivation, leadership, and organizational culture on satisfaction and employee performance. The Journal of Asian Finance, $\begin{array}{llll}\text { Economics, } & \text { and }\end{array}$ https://doi.org/10.13106/JAFEB.2020.VOL7.NO8.577

Patrón-Cortés, R. M., \& Canul, C. A. P. (2017). Compromiso organizacional de los trabajadores: estudio de una empresa refresquera ubicada en México. Revista Electrónica Sobre Cuerpos Académicos y Grupos de Investigación, 4(8).

Peña, M. C., Díaz, M., Chávez, A. G., \& Sánchez, C. E. (2016). El Compromiso Organizacional Como Parte Del Comportamiento De Los Trabajadores De Las Pequeñas Empresas Revista internacional administracion \& finanzas, 9(5), 95105.

Polychroniou, P., \& Trivellas, P. (2018). The impact of strong and balanced organizational cultures on firm performance. International Journal of Quality and Service Sciences, 10(1), 16-35. https://doi.org/10.1108/IJQSS-09-2016-0065

Rahmawati, R., \& Tobing, D. (2019). The Role Of Transformational Leadership On Organizational Commitment And Job Satisfaction Of Tax Office Officers (KPP) In Banjarmasin. International Journal of Scientific \& Technology Research, 8(7), 11-17.

Robbins, S., \& Judge, T. (2009). Comportamiento organizacional. Decimotercera edición.

Sánchez, V. R., \& Franco, F. d. J. M. (2019). Evaluación del compromiso organizacional de Servidores Públicos en México. Revista Arbitrada Interdisciplinaria Koinonía, 4(8), 166-189. http://dx.doi.org/10.35381/r.k.v4i8.263

Schein, E. H. (1986). What you need to know about organizational culture. Training \& Development Journal, 40(1), 30-33.

Segredo, A. M. (2013). Clima organizacional en la gestión del cambio para el desarrollo de la organización. Revista cubana de salud pública, 39, 385-393.

Singh, S. K., \& Singh, A. P. (2019). Interplay of organizational justice, psychological empowerment, organizational citizenship behavior, and job satisfaction in the context of circular economy. Management Decision. https://doi.org/10.1108/MD09-2018-0966 
Spector, P. E. (1985). Measurement of human service staff satisfaction: Development of the Job Satisfaction Survey. American journal of community psychology, 13(6), 693-713. https://doi.org/10.1007/BF00929796

Steckerl, V. (2011). Modelo explicativo de una empresa familiar que relaciona valores del fundador, cultura organizacional y orientación al mercado. Revista científica Pensamiento y Gestión(20).

Tinoco, O., Quispe, C., \& Beltran, V. (2014). Cultura organizacional y satisfacción laboral en la facultad de Ingeniería Industrial en el marco de la acreditación universitaria. Industrial data, 17(2), 56-66.

Vieira, C. (2014). Gestión de recursos humanos: indicadores y herramientas. Observatorio laboral revista Venezolana, 7(14), 23-33.

Wahyu, D. (2013). The relationship between employee engagement, organizational citizenship behavior, and counterproductive work behavior. International Journal of Business Administration, 4(2), 46. http://dx.doi.org/10.5430/ijba.v4n2p46

Yanti, S., \& Dahlan, J. A. (2017). The effects of organizational culture, leadership behavior, and job satisfaction on employee organizational commitment. Journal of Positive Management, 8(4), 80-96. http://dx.doi.org/10.12775/JPM.2017.132

Yu, P.-L. (2017). Innovative culture and professional skills. International Journal of Manpower, 38(2), 198-214. https://doi.org/10.1108/IJM-10-2014-0214

Yusuf, F. A. (2020). The Effect of Organizational Culture on Lecturers' Organizational Commitment in Private Universities in Indonesia. International Journal of Higher Education, 9(2), 16-24. https://doi.org/10.5430/ijhe.v9n2p16 